\title{
COMPACT SETS OF DIVERGENCE FOR CONTINUOUS FUNCTIONS ON A VILENKIN GROUP
}

\author{
DAVID C. HARRIS ${ }^{1}$
}

\begin{abstract}
Let $G$ be a Vilenkin group. Let $E \subset G$ be closed with Haar measure zero. We show there is a continuous function whose Vilenkin-Fourier series diverges at every point in $E$.
\end{abstract}

1. Introduction. Kahane and Katznelson (see [2, Chapter II, 3.4]) have shown that every set of measure zero is a set of divergence for trigonometric-Fourier series of continuous functions. It was expected that the same result would hold for WalshFourier series, but repeated attempts have failed to settle this question. (For a survey of these attempts see [4].) Thus far only particular uncountable sets of divergence have been identified.

In this paper we consider the problem for general Vilenkin groups $G$ and show that any closed subset of $G$ of Haar measure zero is a set of divergence for continuous functions. Our proof, in the spirit of Kahane and Katznelson, depends upon the construction of polynomials $P_{1}, P_{2}, \ldots$ all uniformly bounded by 1 , but having partial sums which are successively larger and larger on the compact set $E$ under question.

For $G$ of bounded type Gosselin $[\mathbf{1}]$ has shown that $E$ must have Haar measure zero. Nevertheless the most general set of divergence remains unknown.

We are very grateful to Dr. William R. Wade for discussions concerning this problem.

2. Basic facts for Vilenkin groups. A Vilenkin group is defined to be a compact, second countable, zero-dimensional abelian group. It follows that there is a chain of subgroups

$$
G=G_{0} \supset G_{1} \supset G_{2} \supset \cdots \supset \bigcap_{k=0}^{\infty} G_{k}=\{0\}
$$

forming a neighborhood base at 0 in $G$ and such that $\left|G_{k} / G_{k+1}\right|=p_{k+1}$ for some primes $p_{k}, k=1,2, \ldots$ Each $G_{k}$ is open and compact. Setting $N_{0}=1$ and $N_{k}=p_{1} \cdot p_{2} \cdot \ldots \cdot p_{k}$ for $k=1,2,3, \ldots$ gives that $\left|G / G_{k}\right|=N_{k}$. It follows that normalized Haar measure $\mu$ on $G$ is given by $\mu\left(G_{k}\right)=N_{k}^{-1}$ for $k=0,1,2, \ldots$

Let $\psi$ be a character of $G$. Then there is $k$ such that $\psi \equiv 1$ on $G_{k}$. The smallest such $k$ is called the conductor of $\psi$. If $\psi$ has conductor $k$, then $\psi$ can be viewed as a character on the finite group $G / G_{k}$. Conversely, a character on $G / G_{k}$ yields

Received by the editors October 10, 1985.

1980 Mathematics Subject Classification (1985 Revision). Primary 42C10; Secondary 43A50, 43A70.

Key words and phrases. Walsh functions, Vilenkin group, sets of divergence.

${ }^{1}$ This research was partially supported by the National Science Foundation under grant INT8400708 . 
a character on $G$ and distinct characters on $G / G_{k}$ yield distinct characters on $G$. Thus there are $N_{k}$ characters with conductor $\leq k$ for $k=0,1,2, \ldots$ The characters can be ordered so that

$$
\begin{array}{ll}
\psi_{0} \equiv 1, & \\
\psi_{l} \text { has conductor } k & \text { for } N_{k-1} \leq l<N_{k}, \quad \text { and } \\
\psi_{N_{k} \cdot j+l}=\psi_{N_{k} \cdot j} \psi_{l} & \text { for } k=0,1,2, \ldots, \\
& \text { for } j=1,2,3, \ldots, \\
& \text { and } l=0,1, \ldots, N_{k}-1 .
\end{array}
$$

The above conditions allow some arbitrariness in the ordering of the characters. Indeed, if one proceeds by induction then the above conditions allow the choice of $\psi_{N_{k} \cdot j}$ for $j=1, \ldots, p_{k}$ to be any character of conductor $k+1$ not already chosen. (More restrictive conditions for ordering the characters are sometimes given, see [3].) Define $m \oplus n$ for nonnegative integers $m$ and $n$ by requiring $\psi_{m} \cdot \psi_{n}=\psi_{m \oplus n}$. We let $\ominus$ be the inverse operation for $\oplus$. The condition $\psi_{N_{k} \cdot j+l}=\psi_{N_{k} \cdot j} \cdot \psi_{l}$ given above becomes $N_{k} \cdot j \oplus l=N_{k} \cdot j+l$ for $l=0,1, \ldots, N_{k}-1$.

Let $\hat{f}(l)=S_{G} f(x) \overline{\psi_{l}(x)} d x$ for any $f \in L^{1}(G)$. Set $S_{m} f(x)=\sum_{l=0}^{m-1} \hat{f}(l) \psi_{l}(x)$ and $S^{*} f(x)=\sup _{m \geq 0}\left|S_{m} f(x)\right|$. The well-known identity

$$
\sum_{l=0}^{N_{k}-1} \psi_{l}(x)= \begin{cases}N_{k}, & x \in G_{k}, \\ 0, & x \in G \sim G_{k}, k=0,1,2, \ldots\end{cases}
$$

implies that

$$
\sum_{l=0}^{N_{k}-1} \psi_{l}(-t) \psi_{l}(x)=\sum_{l=0}^{N_{k}-1} \psi_{l}(x-t)= \begin{cases}N_{k}, & x \in G_{k}+t \\ 0, & x \in G \sim\left(G_{k}+t\right) .\end{cases}
$$

Thus any function $f$ constant on cosets of $G_{k}$ is a $G$-polynomial, i.e. such $f$ equals $\sum_{l=0}^{M} \hat{f}(l) \psi_{l}(x)$ for some $M<\infty$.

\section{The main theorem.}

THEOREM. If $E \subset G$ is compact and has measure 0 , then there is $f \in C(G)$ such that $S_{m} f(x)$ diverges for $x \in E$.

In $\S 5$, we extend the result to $F$ - $\sigma$ sets.

To prove the theorem it suffices to construct polynomials $R_{l}$ for $l=1,2, \ldots$ such that $\left\|R_{l}\right\|_{\infty} \leq 1$ and $S^{*} R_{l}(x) \geq l / 2$ for $x \in E$. Indeed, given such polynomials, then, by induction on $l$, we can choose $k_{l}$ so that

$$
P_{l} \equiv \psi_{N_{k_{l}}} \cdot R_{2^{l}} \text { satisfies } \hat{P}_{l}(j) \hat{P}_{m}(j)=0 \quad \text { when } m<l \text { and for } j=0,1,2, \ldots
$$

It is thus clear that $\sum_{l=1}^{\infty}\left(P_{l}(x) / l^{2}\right)$ converges absolutely and uniformly to a continuous function $f$. Furthermore, the Vilenkin-Fourier series of $f$ is given by

$$
\sum_{l=1}^{\infty} \sum_{j=N_{k}}^{N_{k_{l+1}}-1} \frac{1}{l^{2}} \hat{P}_{l}(j) \psi_{j}(x)
$$


In particular,

$$
\begin{aligned}
S^{*} f(x) & \geq \sup _{l \geq 1} S^{*} \frac{P_{l}(x)}{l^{2}}-\sum_{l=1}^{\infty}\left|\frac{P_{l}(x)}{l^{2}}\right| \\
& \geq \sup _{l \geq 1} S^{*} \frac{R_{2^{l}}(x)}{l^{2}}-\sum_{l=1}^{\infty} \frac{1}{l^{2}} \geq \sup _{l \geq 1} \frac{2^{l-1}}{l^{2}}-\sum_{l=1}^{\infty} \frac{1}{l^{2}}=\infty
\end{aligned}
$$

as desired.

4. Construction of $\left\{R_{l}\right\}$. The construction is given in three steps.

(i) For $i=0$ and 1 , let $H_{i}$ be a finite union of cosets of some $G_{k_{i}}$. For example, $H_{0}=\bigcup_{j=1}^{M} I_{j}$ with $\mu\left(I_{j}\right)=\mu\left(G_{k_{0}}\right)=N_{k_{0}}^{-1}$. Suppose further that $H_{0} \supset H_{1}$ and that

$$
\mu\left(I_{j} \cap H_{1}\right) \leq \mu\left(I_{j} \sim H_{1}\right), \quad \text { for } 1 \leq j \leq M .
$$

Claim. Given any $G$-polynomial $R$ of order less than $N_{k_{0}}$ which satisfies $\|R\|_{\infty} \leq 1, R \equiv 0$ on $H_{0}$ and $\left|S_{m} R(x)\right| \geq l / 2$ for some integers $m, l \in \mathbf{N}$ and all $x \in H_{0}$, there exists a $G$-polynomial $Q$ of order less than $N_{k_{1}}$ which satisfies

$$
\|Q\|_{\infty} \leq 1, \quad|Q| \leq \frac{1}{2} \quad \text { on } H_{0}
$$

and

$$
\left|S_{m+r} Q(x)\right| \geq(l+1) / 2 \text { for all } x \in H_{1} \text { where } r=N_{k_{1}}-N_{k_{0}} .
$$

To prove this claim set

$$
g(x)= \begin{cases}\frac{1}{2} S_{m} R(x) /\left|S_{m} R(x)\right| & \left(S_{m} R(x) \neq 0\right) \\ \frac{1}{2} & \left(S_{m} R(x)=0\right)\end{cases}
$$

Set

$$
\alpha_{j}=\frac{-1}{\left(I_{j} \sim H_{1}\right)} \int_{I_{j} \cap H_{1}} g \quad(1 \leq j \leq M)
$$

and define a function $f$ on $G$ by

$$
f(x)= \begin{cases}g(x), & \left(x \in H_{1}\right), \\ \alpha_{j}, & \left(x \in I_{j} \sim H_{1}, 1 \leq j \leq M\right), \\ 0, & \left(x \in G \sim H_{0}\right) .\end{cases}
$$

By the definition of the $\alpha_{j}$ 's, it is clear that

$$
\frac{1}{\mu\left(I_{j}\right)} \int_{I_{j}} f=0 \quad(1 \leq j \leq M)
$$

Since $f$ vanishes off $H_{0}$, it follows that $f$ has mean zero on all cosets of $G_{k_{0}}$. Consequently, $\hat{f}(j) \equiv 0$ for $0 \leq j<N_{k_{0}}$. On the other hand, since $f$ is constant on all cosets of $G_{k_{1}}$ it is clear that $\hat{f}(j) \equiv 0$ for $j \geq N_{k_{1}}$. Hence $f$ is a $G$ polynomial and $f=\sum_{j=N_{k_{0}}}^{N_{k_{1}}-1} \hat{f}(j) \psi_{j}$. In particular, it follows from the choice of $r$ 
that $S_{m+r}\left(\psi_{r} f\right)=\psi_{r} \cdot f$. Since $\widehat{\psi_{r} \cdot R}(j)=\hat{R}(j \ominus r)$ and $\hat{R}(j) \equiv 0$ for $j \geq N_{k_{0}}$ the choice of $r$ implies that

$$
\begin{aligned}
S_{m+r}\left(\psi_{r} R\right) & \equiv \sum_{j=0}^{m+r-1} \hat{R}(j \ominus r) \psi_{j}=\sum_{j=r}^{m+r-1} \hat{R}(j \ominus r) \psi_{j \ominus r} \psi_{r} \\
& =\psi_{r} \sum_{j=0}^{m-1} \hat{R}(j) \psi_{j} \equiv \psi_{r} S_{m}(R) .
\end{aligned}
$$

Consequently, if $Q=\psi_{r}(f+R)$, then

$$
S_{m+r}(Q)=\psi_{r}\left(f+S_{m}(R)\right) .
$$

To see that $Q$ enjoys the promised properties, let $x \in H_{1}$ and suppose that $S_{m} R(x) \neq 0$. Then the identity above and the definition of $f$ imply that

$$
\left|S_{m+r} Q(x)\right| \equiv\left|f(x)+S_{m} R(x)\right|=\left|\frac{1}{2} S_{m} R(x) /\right| S_{m} R(x)\left|+S_{m} R(x)\right| \geq(l+1) / 2
$$

by (2). On the other hand, if $x \in H_{1}$ but $S_{m} R(x)=0$, then (2) implies $l=0$. Hence

$$
\left|S_{m+r} Q(x)\right| \equiv\left|f(x)+S_{m} R(x)\right|=\frac{1}{2}+0=(l+1) / 2 .
$$

Thus $\left|S_{m+r} Q\right| \geq(l+1) / 2$ on $H_{1}$ in all cases. To obtain bounds for $Q$, observe that the supports of $f$ and $R$ are disjoint by definition. Since both $f$ and $R$ are dominated by 1 , it follows that $\|Q\|_{\infty} \leq 1$. Moreover, (1) implies that $|f| \leq \frac{1}{2}$ on $H_{0}$. Since $R$ vanishes on $H_{0}$, it is clear that $|Q| \leq \frac{1}{2}$ on $H_{0}$. Finally, by construction, $Q$ is a $G$-polynomial of order less than $N_{k_{1}}$. Hence the proof of the claim is complete.

(ii) For the second step, use the fact that $E$ is compact to choose sets $G \equiv E_{0} \supset$ $E_{1} \supset \cdots$ such that $E=\bigcap_{l=1}^{\infty} E_{l}$, and such that $E_{l}$ is a finite union of cosets of some $G_{k(l)}$, say $E_{l} \equiv \bigcup_{j=1}^{M(l)} I_{j}^{(l)}$. Since $\mu(E)=0$ we may suppose that $k(l)<k(l+1)$ and when constructing $E_{l+1}$ from $E_{l}$ that each coset $I_{j}^{(l)}$ has been diminished by at least half, i.e.,

$$
\mu\left(I_{j}^{(l)} \cap E_{l+1}\right) \leq \mu\left(I_{j}^{(l)} \sim E_{l+1}\right) \quad \text { for } 1 \leq j \leq M(l) \text { and } l \in \mathbf{N} .
$$

(iii) For the third step we show by induction that to each $l \in \mathbf{N}$ there corresponds a $G$-polynomial $R_{l}$ and an integer $m(l)$ such that $\left\|R_{l}\right\|_{\infty} \leq 1, R_{l} \equiv 0$ on $E_{2 l}$, and $\left|S_{m(l)} R_{l}(x)\right| \geq l / 2$ for all $x \in E_{2 l}$.

Set $R_{0} \equiv 0$ and suppose that such $R_{l}$ and $m(l)$ have been chosen. Apply the claim to $H_{0}=E_{2 l}, H_{1}=E_{2 l+1}, M=M(2 l), k_{0}=k(2 l), k_{1}=k(2 l+1)$, and $R=R_{l}$. Thus obtain a $G$-polynomial $Q$ such that $\|Q\|_{\infty} \leq 1,|Q| \leq \frac{1}{2}$ on $E_{2 l}$, and order of $Q \leq N_{k(2 l+1)}$, and $\left|S_{m+r} Q(x)\right| \geq(l+1) / 2$ for all $x \in E_{2 l+1}$, where $m=m(l)$ and $r=N_{k(2 l+1)}-N_{k(2 l)}$. Let

$$
\beta_{j}=\frac{1}{\mu\left(I_{j}^{(2 l+1)} \sim E_{2 l+2}\right)} \int_{I_{j}^{(2 l+1)} \cap E_{2 l+2}} Q
$$

for $1 \leq j \leq M(2 l+1)$ and define $h$ on $G$ by

$$
h(x)= \begin{cases}-Q(x), & \left(x \in E_{2 l+2}\right), \\ \beta_{j}, & \left(x \in I_{j}^{(2 l+1)} \sim E_{2 l+2}\right), 1 \leq j \leq M(2 l+1), \\ 0, & \left(x \in[0,1) \sim E_{2 l+1}\right) .\end{cases}
$$


Finally, set $R_{l+1}=h+Q$ and $m(l+1)=m+r$.

Let $x \in E_{2 l}$ and observe by the choice of $Q$ and (3) that $|h(x)| \leq \frac{1}{2}$. Hence $\left|R_{l+1}(x)\right| \leq|h(x)|+|Q(x)| \leq \frac{1}{2}+\frac{1}{2}$. On the other hand, if $x \in[0,1) \sim E_{2 l}$, then $\left|R_{l+1}(x)\right| \equiv|Q(x)| \leq 1$. Consequently, $\left\|R_{l+1}\right\| \leq 1$.

Let $x \in E_{2 l+2}$. By construction, $h(x)=-Q(x)$. Hence it is clear that $R_{l+1}$ vanishes on $E_{2 l+2}$.

To estimate the Vilenkin-Fourier series of $R_{l+1}$, observe by construction that $h$ has mean zero on each coset of $G_{k(2 l+1)}$ and $h$ is constant on each coset of $G_{k(2 l+2)}$. Therefore,

$$
R_{l+1} \equiv \sum_{j=0}^{N_{k(2 l+1)}-1} \hat{Q}(j) \psi_{j}+\sum_{j=N_{k(2 l+1)}}^{N_{k(2 l+2)}-1} \hat{h}(j) \psi_{j} .
$$

Since $m(l+1)<N_{k(2 l+1)}$, we conclude that

$$
\left|S_{m(l+1)} R_{l+1}(x)\right| \equiv\left|S_{m+r} Q(x)\right| \geq(l+1) / 2
$$

for all $x \in E_{2 l+1} \supset E_{2 l+2}$. Step (iii) is finished and thus the theorem is proved.

5. $F$-sigma sets. Let $E_{j}$ for $j=1,2, \ldots$ be compact sets of measure zero. For each $j$ construct $R_{l}=R_{l}^{j}$ as in $\S 4$. Now let $P_{l}^{j}=R_{2}^{j} l / l^{2} j^{2}$ to get a countable collection of polynomials satisfying $\sum_{j, l}\left\|P_{l}^{j}\right\|_{\infty}$ and $\sup _{j, l} S^{*} P_{l}^{j}(x)=\infty$ on $E \equiv$ $\bigcup_{j=1}^{\infty} E_{j}$. Hence $f$ defined by $f=\sum \psi_{N_{k(j, l)}} P_{l}^{j}$ for appropriate $N_{k(l, j)}$ is continuous and will satisfy " $S_{m} f(x)$ diverges unboundedly for all $x$ in $E$ ". Thus any $F-\sigma$ set of measure zero is a set of divergence for $C(G)$.

\section{BIBLIOGRAPHY}

1. J. Gosselin, Almost everywhere convergence of Vilenkin-Fourier series, Trans. Amer. Math. Soc. 185 (1973), 345-370.

2. Y. Katznelson, An introduction to harmonic analysis, Dover, New York, 1976.

3. N. Ja. Vilenkin, On a class of complete orthogonal systems, Izv. Akad. Nauk SSSR Ser. Mat. 11 (1947), 363-400; English transl., Amer. Math. Soc. Transl. (2) 28 (1963), 1-35.

4. W. R. Wade, Recent developments in the theory of Walsh series, Internat. J. Math \& Math Sci. 5 (1982), 625-673.

Department of Mathematics, University of Tennessee, KNoxville, TenNESSEE 37996 